\title{
Parameter estimates for two-way repeated measurement MANOVA based on multivariate Laplace distribution
}

\author{
Müge Borazan Çelikbıçak (iD*1, Serpil Aktaş Altunay (iD)2 \\ ${ }^{1}$ Department of Security Sciences, Gendarmerie and Coast Guard Academy, Ankara, Turkey \\ ${ }^{2}$ Department of Statistics, Hacettepe University, Ankara, Turkey
}

\begin{abstract}
Repeated measures data describe multiple measurements taken from the same experimental unit under the different treatment conditions. In particular, researches with repeated measures data in various fields such as health and behavioral sciences, education, and psychology have an important role in applied statistics. There are many methods used to analyze the results of research designs planned with these measurements. The most important difference between these methods is the assumptions on which the models are based. One of the most important assumptions needed by classical methods is the normality assumption. Many methods are valid under the assumption of normality. However, it is not always possible to hold this assumption in applications. For this reason, in the analysis of repeated measures data, different distributions are necessary that can provide flexibility beyond the normal distribution, especially in cases where the assumption of normality does not hold. In this study, it is proposed to use Multivariate Laplace distribution (MLD) which is an alternative distribution in cases where normality assumption does not hold by examining the multivariate variance analysis model (MANOVA). Under MLD assumption, the parameter estimates for the Two-way Repeated Measures MANOVA model are carried out with the maximum likelihood (ML) estimation and ML estimates are obtained via the EM Algorithm.
\end{abstract}

Mathematics Subject Classification (2010). 62H10, 62H12, 62J10, 62K25

Keywords. MANOVA, repeated measures data, multivariate Laplace distribution, EM algorithm, repeated measures MANOVA

\section{Introduction}

In order to build a statistical model, providing data with the default distribution conditions is a very important part of the statistical inferences. Therefore, normal distribution plays an essential role in statistical data analysis. Many of the inferential statistical methods for multivariate data have also been developed for data with Multivariate Normal

\footnotetext{
${ }^{*}$ Corresponding Author.

Email addresses: muge.borazancelikbicak@jsga.edu.tr (M. Borazan Çelikbıçak), spxl@hacettepe.edu.tr (S. Aktaş Altunay)

Received: 27.05.2020; Accepted: 13.07.2020
} 
distribution as a basic assumption. Taking this assumption into consideration, the Analysis of Variance (ANOVA) model is made with the Ordinary Least Squares (OLS) method, which is one of the most frequently used methods for estimating parameters [8]. The estimators obtained with these methods are the most effective estimators with the assumption of normality. In the absence of normality assumption, OLS estimators lose their effectiveness [8]. Therefore, $\mathrm{F}$ test statistics based on OLS estimators are also lose their power. However, in real-life applications, the majority of data sets dont not exactly follow the multivariate normal distribution. Accordingly, it will be more useful to develop and use alternative multivariate distribution methods in cases where the multivariate normality assumption is not provided in order to overcome similar problems.

For MANOVA, assumption of Multivariate Normal distribution is the fundamental distribution as the basic principle. In practice, however, numerous multivariate distributions have been used in the literature to provide solutions to situations where the assumptions of this distribution are not valid. Many studies have made statistical inferences using Elliptically Contoured distributions and similar distributions alternative to normal distribution $[1,22,29]$. Distributions in this context have been found to be more flexible and adaptable particularly for marginal distribution with longer tails. In the Elliptically Contoured distributions, linear and quadratic functions of observations have been shown to be more durable than methods under the assumption of normality [16].

It has been conclusively demonstrated that distributions with heavier tails than the normal distribution (heavy-tailed) are more sensitive to outliers and errors than statistical inferences based on the Normal distribution. Thus, the fact that more flexible distributions are created as an alternative to Normal distribution in order to model data sets containing errors with heavier tails than the Normal distribuiton has emerged. The heavy-tailed distributions recommended as an alternative to the normal distribution are the $t$ and slash distributions [4]. Both distributions can be obtained as a mixture of the non-negative random variable scale and the random variable with a Normal distribution. In this case, both distributions belong to the family of distributions consisting of the scale mixture of the normal distribution.

Another distribution that is very useful in modeling when normality conditions are not met is the Kotz-type distribution proposed by Kotz in 1975 [27]. This distribution can be considered as the generalized version of the Normal distribution. It is possible to write the Kotz-type distribution in terms of scale mixed distribution in order to produce a distribution with heavier tails like the scale mixed distribution of the normal distribution. The scale mixed Kotz-type distribution to obtain the Generalized Multivariate Slash distribution has been proposed as another distribution family that gives better results than other alternative methods in cases where the normal distribution is not followed by data [5]. The aim of this study is to obtain parameter estimates for the Two-way Repeated Measures ANOVA with MANOVA approach using the MLD which is an alternative distribution to Normal distribution in cases where normality assumptions are not met.

The rest of the article is organized as follows: In the following section, brief information about the Repeated Measures Data and properties of Repeated Measures and Two-way Repeated Measures MANOVA model are described. In Section 3, the MLD is given in detail. In the fourth section, estimation of Two-way Repeated Measures MANOVA model parameters are obtained under the proposed distribution by using the ML estimation methodology. Using the Euclidian distance criterion, the effectiveness of the parameter estimates obtained as a result of theoretical inferences examined via a simulation study in Section 5. A real dataset is analyzed to show the implementation of the proposed methodologies in Section 6. Concluding remarks are given in the last section. 
Table 1. Repeated Measures Data Layout

\begin{tabular}{|c|c|c|c|c|c|c|}
\hline Group & Subject & 1 & $\ldots$ & $\mathrm{k}$ & $\ldots$ & $\mathrm{t}$ \\
\hline \multirow{5}{*}{1} & 1 & $y_{111}$ & $\ldots$ & $y_{11 k}$ & $\ldots$ & $y_{11 t}$ \\
\hline & $\vdots$ & $\vdots$ & $\vdots$ & $\vdots$ & $\vdots$ & $\vdots$ \\
\hline & $\mathrm{j}$ & $y_{1 j 1}$ & $\ldots$ & $y_{1 j k}$ & $\ldots$ & $y_{1 j t}$ \\
\hline & $\vdots$ & $\vdots$ & $\vdots$ & $\vdots$ & $\vdots$ & $\vdots$ \\
\hline & $n_{1}$ & $y_{1 n_{1} 1}$ & $\ldots$ & $y_{1 n_{j} k}$ & $\ldots$ & $y_{1 n_{1} t}$ \\
\hline \multirow{5}{*}{2} & 1 & $y_{211}$ & $\ldots$ & $y_{21 k}$ & $\ldots$ & $y_{21 t}$ \\
\hline & $\vdots$ & $\vdots$ & $\vdots$ & $\vdots$ & $\vdots$ & $\vdots$ \\
\hline & $\mathrm{j}$ & $y_{2 j 1}$ & $\ldots$ & $y_{2 j k}$ & $\ldots$ & $y_{2 j t}$ \\
\hline & $\vdots$ & $\vdots$ & $\vdots$ & $\vdots$ & $\vdots$ & $\vdots$ \\
\hline & $n_{2}$ & $y_{2 n_{1} 1}$ & $\ldots$ & $y_{2 n_{j} k}$ & $\ldots$ & $y_{2 n_{1} t}$ \\
\hline$\vdots$ & $\vdots$ & $\vdots$ & $\vdots$ & $\vdots$ & $\vdots$ & $\vdots$ \\
\hline \multirow{5}{*}{$\mathrm{i}$} & 1 & $y_{i 11}$ & $\ldots$ & $y_{i 1 k}$ & $\ldots$ & $y_{i 1 t}$ \\
\hline & $\vdots$ & & $\vdots$ & $\vdots$ & $\vdots$ & $\vdots$ \\
\hline & 1 & $y_{i j 1}$ & $\cdots$ & $y_{i j k}$ & $\cdots$ & $y_{i j t}$ \\
\hline & $\vdots$ & $\vdots$ & $\vdots$ & $\vdots$ & $\vdots$ & $\vdots$ \\
\hline & $n_{i}$ & $y_{2 n_{i} 1}$ & $\ldots$ & $y_{2 n_{i} k}$ & $\ldots$ & $y_{2 n_{i} t}$ \\
\hline$\vdots$ & $\vdots$ & $\vdots$ & $\vdots$ & $\vdots$ & $\vdots$ & $\vdots$ \\
\hline \multirow{5}{*}{$\mathrm{g}$} & 1 & $y_{g 11}$ & $\ldots$ & $y_{g 1 k}$ & $\ldots$ & $y_{g 1 t}$ \\
\hline & $\vdots$ & $\vdots$ & $\vdots$ & $\vdots$ & $\vdots$ & $\vdots$ \\
\hline & $\mathrm{j}$ & $y_{g j 1}$ & $\cdots$ & $y_{g j k}$ & $\cdots$ & $y_{g j t}$ \\
\hline & $\vdots$ & $\vdots$ & $\vdots$ & $\vdots$ & $\vdots$ & $\vdots$ \\
\hline & $n_{g}$ & $y_{g n_{1} 1}$ & $\ldots$ & $y_{g n_{j} k}$ & $\cdots$ & $y_{g n_{1} t}$ \\
\hline
\end{tabular}

\section{Repeated measures data structure}

The term "Repeated Measures" includes univariate or multivariate responses obtained from each experimental unit or subject in multiple cases or under multiple conditions. The term Longitudinal Data is also frequently used to describe repeatedly measured data. Although there have been many approaches to the analysis of repeated measures data, most are limited to situations where the response variables are normally distributed and the data is balanced and complete.

Table 1 illustrates a data set of repeated measures taken from $n_{i}$ th subjects in $t$ th group at time point (in measurement cases) [9]. Where, $n$ indicates the number of independent experimental units or subjects from which repeated measures are obtained; $n_{i}$, indicates the number of subjects or experimental units in each group, and $t$, the number of situations where repeated measures are taken. Also, $y_{i j k}$ denotes the response variable for the measurement taken at the time point $k$ the $i$ th group and $j$ th subject (experimental unit) where $i=1, \ldots, g ; j=1, \ldots n_{i} ; k=1, \ldots t ; n=\sum_{i=1}^{g} n_{i}$.

The main advantage of these studies in which repeated measures are obtained from each subject is that this is the only design type in which it is possible to obtain information 
about individual change models. Such a design is also economical for situations where the decision regarding how to use subjects is made beforehand. As the sources of variation between subjects can be excluded from the experimental error, repeated measures designs often provide more efficient estimators of the relevant parameters than cross-sectional designs in the same number and model. In addition, it is assumed that data can be collected more reliably in a study where the same subjects are repeatedly followed according to a cross-sectional study [9].

The Two-way Repeated Measures ANOVA model is considered to analyze the data structure given in Table 1 as follows,

$$
y_{i j k}=\mu+\gamma_{i}+(\gamma \tau)_{i k}+\varepsilon_{i j k}
$$

where, $y_{i j k}$ is the response variable corresponding to the $j t h$ observation in the $i t h$ group and $k$ th treatment; $\mu$ is the overall mean; $\tau_{k}$ is the effect of $k t h$ treatment; $y_{i}$ is the effect of $i t h$ group; $(\gamma \tau)_{i k}$ is the interaction effect between $k t h$ treatment and $i t h$ group and $\varepsilon_{i j k}$ are the independently and identically distributed (i.i.d.) error terms.

There is a considerable amount of literature examining MANOVA and Multivariate Linear Models for repeated measures. [9,13,17,20,21,26,28]. Within the scope of MANOVA, the General Linear Model approach will be used for representation of multivariate repeated measures. In general, a Multivariate Linear Model is shown as follows,

$$
\boldsymbol{Y}=\boldsymbol{X} \boldsymbol{\beta}+\boldsymbol{E}
$$

where, $\boldsymbol{Y}$ is the $(g x 1)$ dimensional response variable vector; $\boldsymbol{X},(g x(k+1))$ dimensional design matrix ; $\boldsymbol{\beta}$ is the $((k+1) x 1)$ dimensional parameter vector and $\boldsymbol{E}$ is $(g x 1)$ dimensional error vector. In the linear model (2.2), the parameters of Two-way Repated measures ANOVA model (2.1) with will be included in the $\boldsymbol{\beta}$ coefficients. In this context, Eq. (2.2) is written with data indices as follows,

$$
\boldsymbol{Y}_{i j}=\boldsymbol{X}_{i} \boldsymbol{\beta}+\varepsilon_{i j}
$$

where, $\boldsymbol{Y}_{i j}^{T}=\left(y_{111}, y_{112}, \ldots, y_{1 n_{1} t}, \ldots, y_{g n_{i} 1}, y_{g n_{i} 2}, \ldots, y_{g n_{i} t}\right), \boldsymbol{X}_{i}$ is design matrix depending on the number of groups consisting of zeros and ones and $\boldsymbol{\beta}^{T}=\left(\mu, \tau_{1}, \tau_{2}, \ldots, \tau_{t}, \gamma_{1}, \ldots, \gamma_{g}\right.$, $\left.(\gamma \tau)_{11}, \ldots,(\gamma \tau)_{t g}\right)$. In our study, we assume that the distribution of error terms in the linear model (2.3) follows MLD with $\boldsymbol{\varepsilon}_{i j} \sim M L_{p}\left(\mathbf{0}, \boldsymbol{\Sigma}_{p}\right)$.

\section{Multivariate Laplace distribution}

The MLD appears in many applications, especially in the fields of finance and biological sciences as well as the modeling of current life data. The advantages of the Laplace distribution, although having tails thicker than normal, can be listed as the presence of moments, simple relationships between distribution parameters and moments, and a simple representation of the characteristic function. The distribution is defined by two multivariate parameters: one is the scale parameter, and the other is the location parameter, which simultaneously controls both the form and the location of the distribution. The MLD appears in the literature with its use in various forms and in different fields $[2,12,19,23,25,30,31]$. The Multivariate Symmetric Laplace distribution has been defined as the generalized of the MLD [18]. In this study, the Multivariate Symmetric Laplace distribution is used which is generalization of the Univariate Laplace distribution when the skewness parameter taken zero in the Multivariate Skewed Laplace distribution proposed by [3]. Probability density function related to Multivariate Symmetrical Laplace distribution is given as,

$$
f_{M L D}(\boldsymbol{y} ; \boldsymbol{\mu}, \boldsymbol{\Sigma})=\frac{|\boldsymbol{\Sigma}|^{-1 / 2}}{2^{p} \pi^{(p-1) / 2} \Gamma\left(\frac{p+1}{2}\right)} e^{-\sqrt{(\mathbf{y}-\boldsymbol{\mu})^{T} \boldsymbol{\Sigma}^{-1}(\mathbf{y}-\boldsymbol{\mu})}}
$$


where, $\boldsymbol{Y} \epsilon R^{p}, p \geq 1$ and $\boldsymbol{\mu} \epsilon R^{p}$ denotes the location vector, $\boldsymbol{\Sigma}$ is a positive definite scatter matrix. In Eq.(3.1), for $p=1$, it can be obtained Univariate Laplace distribution function and this distribution is considered as a multivariate extension of the Univariate Laplace distribution [3]. The mean, variance, skewness and kurtosis of this distribution are given respectively,

$$
E(\boldsymbol{Y})=\boldsymbol{\mu}, \quad \operatorname{Var}(\boldsymbol{Y})=(p+1) \boldsymbol{\Sigma}, \quad \beta_{1 p}=0, \quad \beta_{2 p}=\frac{p(p+2)(p+3)}{(p+1)}
$$

$[3,29]$. One of the most important features of the Laplace distribution is that it can be characterized by other probability distributions [18]. The other feature of the Laplace distribution is that it can be expressed as a scale mixture of Normal Distribution [15]. Taking advantage of this feature, in order to get the the parameter estimates, the variable $\boldsymbol{Y}$ is defined as the scale mixture of the normal distribution as follows,

$$
\boldsymbol{Y}=\boldsymbol{\mu}+\sqrt{\boldsymbol{V}^{-1}} \boldsymbol{\Sigma}^{\frac{1}{2}} \boldsymbol{Z}
$$

where, $\boldsymbol{Z} \sim N_{p}\left(\mathbf{0}, \boldsymbol{I}_{p}\right)$ and $\boldsymbol{V}$ follows the Inverse Gamma distribution with $\boldsymbol{V} \sim I G((p+$ 1)/2,1/2). Henceforth the $\boldsymbol{Y}$ follows the MLD [3]. Defining the $\boldsymbol{Y}$ variable as the scale distribution of the normal distribution makes easier to obtain the ML estimates as the parameters with the EM algorithm. For this purpose, firstly, the combined probability density functions of variable $Y$ and mixed variable $\boldsymbol{V}$ must be obtained via joint probability density functions of the variable with the $\boldsymbol{Z}$ Standard Normal distribution and the variable $\boldsymbol{V}$ with the Inverse Gamma distribution. Thus, we obtain the following joint pdf of $\boldsymbol{Y}$ and $\boldsymbol{V}$ with the following equation,

$$
f(\boldsymbol{Y}, v)=\frac{|\boldsymbol{\Sigma}|^{-1 / 2}}{2 \pi^{p / 2} 2^{(p+1) / 2} \Gamma\left(\frac{p+1}{2}\right)} v^{-3 / 2} e^{-\frac{1}{2}}\left[v^{-1}+v\left((\boldsymbol{Y}-\boldsymbol{X} \boldsymbol{\beta})^{T} \boldsymbol{\Sigma}^{-1}(\boldsymbol{Y}-\boldsymbol{X} \boldsymbol{\beta})\right] .\right.
$$

At this stage, conditional distribution and conditional expectation are necessary to reach the ML estimates via the EM algorithm. Therefore, when $\boldsymbol{Y}$ is given, we obtained the conditional probability distribution of the $\boldsymbol{V}$ variable as follows

$$
f(\boldsymbol{V} \mid \boldsymbol{Y})=\frac{1}{\sqrt{2 \pi}} e^{\sqrt{(\boldsymbol{Y}-\boldsymbol{X} \boldsymbol{\beta})^{T} \boldsymbol{\Sigma}^{-1}(\boldsymbol{Y}-\boldsymbol{X} \boldsymbol{\beta})}} v^{-3 / 2} e^{-\frac{1}{2}\left[v^{-1}+v\left[(\boldsymbol{Y}-\boldsymbol{X} \boldsymbol{\beta})^{T} \boldsymbol{\Sigma}^{-1}(\boldsymbol{Y}-\boldsymbol{X} \boldsymbol{\beta})\right]\right]} .
$$

Using the conditional density function given in Eq.(3.5) we obtain following conditional expectation as

$$
E(\boldsymbol{V} \mid \boldsymbol{Y}, \hat{\boldsymbol{\beta}}, \hat{\boldsymbol{\Sigma}})=\frac{1}{\sqrt{(\boldsymbol{Y}-\boldsymbol{X} \hat{\boldsymbol{\beta}})^{T}(\boldsymbol{Y}-\boldsymbol{X} \hat{\boldsymbol{\beta}})}} .
$$

\section{Parameter estimation with ML}

The iteratively-reweighted algorithm which can be identified as an EM algorithm can be used to obtain the ML estimates. In the following paragraph, we will describe the EM Algorithm to obtain the ML estimates of Repeated Measures MANOVA model parameters. Let $y_{1}, y_{2},, y_{n}$ be a p-dimensional random sample from the MLD with the parameters $\boldsymbol{\mu}$ and $\boldsymbol{\Sigma}$. For using EM algorithm to compute ML estimates we assume that $\boldsymbol{V}$ is missing in the scale mixture representation of the MLD given in Eq. (3.3). We can carry out the EM algorithm as follows [3,24]. Using the conditional distribution given in Eq. (3.5) we obtain the complete data log-likelihood function with the following equation,

$$
\ln L=-\frac{n}{2} \ln |\boldsymbol{\Sigma}|-\frac{1}{2} \sum_{i=1}^{n} v_{i}\left(\boldsymbol{Y}_{i}-\boldsymbol{X}_{i} \boldsymbol{\beta}\right)^{T} \boldsymbol{\Sigma}^{-1}\left(\boldsymbol{Y}_{i}-\boldsymbol{X}_{i} \boldsymbol{\beta}\right)-\frac{1}{2} \sum_{i=1}^{n}\left(3 \ln \left(v_{i}\right)+\left(v_{i}\right)^{-1}\right) .
$$

It is easier to maximize this function than unknown parameters $[3,7,10]$. However, the conditional expected value of the log-likelihood function for the given any observed data 
Table 2. Steps of the EM Algorithm for $\boldsymbol{\beta}$ and $\boldsymbol{\Sigma}$ estimates

1. Set the iteration number $k=1$ and initial values $\boldsymbol{\beta}^{(0)}, \boldsymbol{\Sigma}^{(0)}$.

2. E-Step: Use $\boldsymbol{\beta}^{(k)}, \boldsymbol{\Sigma}^{(k)}$ current estimates and for $k=1,2,3, \ldots$ iteration the weights $w_{i j}^{(k)}$ and also $\sum_{i=1}^{g} \sum_{j=1}^{n_{i}} w_{i j}$ for $i=1, \ldots, g ; j=1, \ldots, n_{i}$.

3. M-Step: Use the new values obtained in E-Step then $\boldsymbol{\beta}^{(k+1)}, \boldsymbol{\Sigma}^{(k+1)}$ with following equations:

$$
\begin{aligned}
& \boldsymbol{\beta}^{(k+1)}=\frac{\sum_{i=1}^{g} \sum_{j=1}^{n_{i}} w_{i j}^{(k)}\left(\boldsymbol{X}_{i}^{T} \boldsymbol{\Sigma}^{(k)^{-1}} \boldsymbol{X}_{i}\right)^{-1}\left(\boldsymbol{X}_{i}^{T} \boldsymbol{\Sigma}^{(k)-1} \boldsymbol{Y}_{i j}\right)}{\sum_{i=1}^{g} \sum_{j=1}^{n_{i}} w_{i j}^{(k)}} \\
& \boldsymbol{\Sigma}^{(k+1)}=\sum_{i=1}^{g} \sum_{j=1}^{n_{i}} w_{i j}^{(k)}\left(\boldsymbol{Y}_{i j}-\boldsymbol{X}_{i} \boldsymbol{\beta}^{(k+1)}\right)\left(\boldsymbol{Y}_{i j}-\boldsymbol{X}_{i} \boldsymbol{\beta}^{(k+1)}\right)
\end{aligned}
$$

4. Repeat $\mathrm{E}$ and $\mathrm{M}$ steps until the convergence rule is obtained.

$\boldsymbol{Y}_{i}$ in Eq. (4.1) must be taken in order to eliminate the missing variable problem in the function. The conditional expected value of Eq. (4.1) as follows,

$$
E\left(\ln L(\boldsymbol{\beta}, \boldsymbol{\Sigma}) \mid \boldsymbol{Y}_{i}, \hat{\boldsymbol{\beta}}, \hat{\boldsymbol{\Sigma}}\right)=-\frac{n}{2} \ln |\boldsymbol{\Sigma}|-\frac{1}{2} \sum_{i=1}^{n} E\left(\boldsymbol{V}_{i} \mid \boldsymbol{Y}_{i}, \hat{\boldsymbol{\beta}}, \hat{\boldsymbol{\Sigma}}\right)\left(\boldsymbol{Y}_{i}-\boldsymbol{X}_{i} \boldsymbol{\beta}\right)^{T} \boldsymbol{\Sigma}^{-1}\left(\boldsymbol{Y}_{i}-\boldsymbol{X}_{i} \boldsymbol{\beta}\right)
$$

where, the conditional expectation $E\left(\boldsymbol{V}_{i} \mid \boldsymbol{Y}_{i}, \hat{\boldsymbol{\beta}}, \hat{\boldsymbol{\Sigma}}\right)$ is given in Eq. (3.6). Therefore, the log-likelihood function of MLD with Repeated Measures MANOVA model in Eq.(2.3) is given by,

$$
Q\left((\boldsymbol{\beta}, \boldsymbol{\Sigma}) \mid \boldsymbol{Y}_{i j}, \hat{\boldsymbol{\beta}}, \hat{\boldsymbol{\Sigma}}\right)=-\frac{n}{2} \ln |\boldsymbol{\Sigma}|-\sum_{i=1}^{g} \sum_{i=1}^{n_{i}} w_{i j}\left(\boldsymbol{Y}_{i j}-\boldsymbol{X}_{i} \boldsymbol{\beta}\right)^{T} \boldsymbol{\Sigma}^{-1}\left(\boldsymbol{Y}_{i j}-\boldsymbol{X}_{i} \boldsymbol{\beta}\right) .
$$

where, $w_{i j}=E\left(\boldsymbol{V}_{i} \mid \boldsymbol{Y}_{i}, \hat{\boldsymbol{\beta}}, \hat{\boldsymbol{\Sigma}}\right)$. To find the ML estimates, the likelihood function is differentiated with respect to the parametres and we obtain the following equations as

$$
\begin{gathered}
\frac{\left.\partial Q\left((\boldsymbol{\beta}, \boldsymbol{\Sigma}) \mid \mathbf{Y}_{i j}, \hat{\boldsymbol{\beta}}, \hat{\boldsymbol{\Sigma}}\right)\right)}{\partial \boldsymbol{\beta}}=\sum_{i=1}^{g} \sum_{j=1}^{n_{i}} w_{i j}\left(-2 \boldsymbol{X}_{i}^{T} \boldsymbol{\Sigma}^{-1} \boldsymbol{Y}_{i j}+2 \boldsymbol{X}_{i}^{T} \boldsymbol{\Sigma}^{-1} \boldsymbol{X}_{i} \boldsymbol{\beta}\right)=0, \\
\frac{\left.\partial Q\left((\boldsymbol{\beta}, \boldsymbol{\Sigma}) \mid \boldsymbol{Y}_{i j}, \hat{\boldsymbol{\beta}}, \hat{\boldsymbol{\Sigma}}\right)\right)}{\partial \boldsymbol{\Sigma}^{-1}}=2\left[\boldsymbol{\Sigma}-\frac{1}{n} \sum_{i=1}^{g} \sum_{j=1}^{n_{i}} w_{i j} \boldsymbol{\Upsilon}_{i j}\right]-\operatorname{diag}\left[\boldsymbol{\Sigma}-\frac{1}{n} \sum_{i=1}^{g} \sum_{j=1}^{n_{i}} w_{i j} \boldsymbol{\Upsilon}_{i j}\right]=0
\end{gathered}
$$

where, $\boldsymbol{\Upsilon}_{i j}=\left(\boldsymbol{Y}_{i j}-\boldsymbol{X}_{i} \boldsymbol{\beta}\right)\left(\boldsymbol{Y}_{i j}-\boldsymbol{X}_{i} \boldsymbol{\beta}\right)^{T}[6]$. The Estimating Equations for $\boldsymbol{\beta}$ which include the parameters of the Two-way Repeated Measures ANOVA model in the linear model expressed in Eq. (2.1) and scatter matrix $\boldsymbol{\Sigma}$ will be as follows,

$$
\begin{gathered}
\hat{\boldsymbol{\beta}}=\frac{\sum_{i=1}^{g} \sum_{j=1}^{n_{i}} w_{i j}\left(\boldsymbol{X}_{i}^{T} \boldsymbol{\Sigma}^{-1} \boldsymbol{X}_{i}\right)^{-1}\left(\boldsymbol{X}_{i}^{T} \boldsymbol{\Sigma}^{-1} \boldsymbol{Y}_{i j}\right)}{\sum_{i=1}^{g} \sum_{j=1}^{n_{i}} w_{i j}}, \\
\hat{\boldsymbol{\Sigma}}=\sum_{i=1}^{g} \sum_{j=1}^{n_{i}} w_{i j}\left(\boldsymbol{Y}_{i j}-\boldsymbol{X}_{i} \boldsymbol{\beta}\right)\left(\boldsymbol{Y}_{i j}-\boldsymbol{X}_{i} \boldsymbol{\beta}\right)^{T}
\end{gathered}
$$

[6]. The steps of EM algorithm to compute the Eq. (4.6) and Eq. (4.7) can be given in Table 2 . 
Table 3. Simulation Parameters

\begin{tabular}{lll}
\hline \hline & & \\
1. & Sample size $n=\sum_{i=1}^{g} n_{i}$ & $20,60,100,140,200$ \\
2. & Number of group $(i=1, \ldots g)$ & 2,3 \\
3. Number of treatment $(k=1, \ldots t)$ & $3,4,6$ \\
4. Number of subject in each treatment $\left(j=1, \ldots, n_{i}\right)$ & $10,30,50,70,100$ \\
$\mathbf{5 .}$ & Replication & 1000 \\
\hline \hline
\end{tabular}

\section{Simulation study}

In this section, a simulation study is performed in $\mathrm{R}$ statistical software to estimate the Two-way Repeated Measures ANOVA model parameters [6]. Numerical values used in the simulation for repeated measurement data structure are given in Table 3.

In the simulation study, the initial values for $\boldsymbol{\beta}$ vector to generate the data are chosen so that they satisfy the restrictions of the fixed-effect Two-way Repeated Measures ANOVA model given in Eq.(2.1). The initial value for the scatter matrix $\boldsymbol{\Sigma}$ is chosen according to the dependency structure among the experiments, providing that the variances of the variables are homogeneous. The EM algorithm is used to calculate the ML estimates of the parameters and OLS estimators of the parameters are taken as the initial values in the algorithm. The Euclidian distance between the estimates and current estimates are calculated to show the effectiveness of parameter estimates. The value of $10^{-6}$ is assigned as the stopping rule and chosen as the iteration convergence criterion and the Euclidian distances is calculated by the following formulas [7]:

$$
\left\|\hat{\boldsymbol{\beta}}^{k+1}-\hat{\boldsymbol{\beta}}^{k}\right\| \leq \Delta=10^{-6}, \quad\left\|\hat{\boldsymbol{\Sigma}}^{k+1}-\hat{\boldsymbol{\Sigma}}^{k}\right\| \leq \Delta=10^{-6}
$$

Moreover, the Euclidian distances are calculated over the log-likelihood functions as follows $[10,11]$,

$$
\left\|\frac{\hat{\mathbf{Q}}^{k+1}}{\hat{\mathbf{Q}}^{k}}-1\right\| \leq \Delta=10^{-6}
$$

The following six scenarios are run in simulation with the algorithm given in Table 4 and the steps of the simulation study are specified in below.

Table 4. Algorithm for Generating Multivariate Repeated Measurement Data with MLD

1. $n \leftarrow$ set the sampling size $(20,60,100,140,200)$.

2. $\boldsymbol{\mu} ; \boldsymbol{\Sigma} ; \boldsymbol{\beta} \leftarrow$ set the initial values.

3. $\boldsymbol{Z} \sim N\left(\mathbf{0}, \boldsymbol{I}_{p}\right) \leftarrow$ generate data from Multivariate Normal Distribution.

4. $\boldsymbol{V} \sim I G\left(\frac{p+1}{2}, \frac{1}{2}\right) \leftarrow$ generate data Inverse Gamma Distribution.

5. $\boldsymbol{\varepsilon}_{i j} \sim \boldsymbol{\mu}+\sqrt{\mathbf{V}^{-1}} \boldsymbol{\Sigma}^{1 / 2} \boldsymbol{Z} \leftarrow$ generate the error terms from MLD.

6. $\boldsymbol{Y}_{i j}=\boldsymbol{X}_{i} \boldsymbol{\beta}+\varepsilon_{i j} \leftarrow$ generate the response variable via Eq.(2.3). 
Scenario $\quad 1: y_{i j k}=\mu+\gamma_{i}+(\gamma \tau)_{i k}+\varepsilon_{i j k} i=1,2 ; k=1,2,3 ; j=1, \ldots, n_{i}$ Initial values;

$$
\begin{aligned}
& \boldsymbol{\beta}^{T}=\left[\begin{array}{llllllllllll}
1 & 1 & 0 & -1 & -1 & 0 & -1 & -1 & 0 & 1 & 1 & 0
\end{array}\right] \\
& \boldsymbol{\Sigma}=\left[\begin{array}{lll}
1.0 & 0.8 & 0.7 \\
0.8 & 1.0 & 0.6 \\
0.7 & 0.6 & 1.0
\end{array}\right]
\end{aligned}
$$

Scenario $\quad 2: y_{i j k}=\mu+\gamma_{i}+(\gamma \tau)_{i k}+\varepsilon_{i j k} i=1,2 ; k=1,2,3,4 ; j=1, \ldots, n_{i}$ Initial values;

$$
\begin{aligned}
& \boldsymbol{\beta}^{T}=\left[\begin{array}{lllllllllllllll}
1 & 1 & 0 & 1 & 1 & 1 & 0 & 1 & 1 & 1 & 0 & -1 & -1 & -1 & 0
\end{array}\right] \\
& \boldsymbol{\Sigma}=\left[\begin{array}{lllll}
1.0 & 0.8 & 0.7 & 0.8 \\
0.8 & 1.0 & 0.6 & 0.7 \\
0.7 & 0.6 & 1.0 & 0.8 \\
0.8 & 0.7 & 0.8 & 1.0
\end{array}\right]
\end{aligned}
$$

Scenario $\quad 3: y_{i j k}=\mu+\gamma_{i}+(\gamma \tau)_{i k}+\varepsilon_{i j k} i=1,2 ; k=1,2,3,4,5,6 ; j=1, \ldots, n_{i}$ Initial values;

$$
\begin{aligned}
& \boldsymbol{\beta}^{T}=\left[\begin{array}{lllllllllllllllllllll}
1 & 1 & 0 & 1 & 1 & 1 & 1 & 1 & 0 & 1 & 1 & 1 & 1 & 1 & 0 & -1 & -1 & -1 & -1 & -1 & 0
\end{array}\right] \\
& \boldsymbol{\Sigma}=\left[\begin{array}{llllll}
1.0 & 0.8 & 0.7 & 0.8 & 0.7 & 0.8 \\
0.8 & 1.0 & 0.6 & 0.7 & 0.6 & 0.7 \\
0.7 & 0.6 & 1.0 & 0.8 & 0.7 & 0.6 \\
0.8 & 0.7 & 0.8 & 1.0 & 0.6 & 0.7 \\
0.7 & 0.6 & 0.7 & 0.6 & 1.0 & 0.7 \\
0.8 & 0.7 & 0.6 & 0.7 & 0.7 & 1.0
\end{array}\right]
\end{aligned}
$$

Scenario $\quad 4: y_{i j k}=\mu+\gamma_{i}+(\gamma \tau)_{i k}+\varepsilon_{i j k} i=1,2,3 ; k=1,2,3 ; j=1, \ldots, n_{i}$

Initial values;

$$
\begin{gathered}
\boldsymbol{\beta}^{T}=\left[\begin{array}{llllllllllllllll}
1 & 1 & 1 & 0 & 1 & 1 & 0 & 1 & 1 & 0 & 1 & 1 & 0 & 0 & 0 & 0
\end{array}\right] \\
\boldsymbol{\Sigma}=\left[\begin{array}{llll}
1.0 & 0.8 & 0.7 \\
0.8 & 1.0 & 0.6 \\
0.7 & 0.6 & 1.0
\end{array}\right]
\end{gathered}
$$

Scenario $\quad \mathbf{5}: y_{i j k}=\mu+\gamma_{i}+(\gamma \tau)_{i k}+\varepsilon_{i j k} i=1,2,3 ; k=1,2,3,4 ; j=1, \ldots, n_{i}$ Initial values;

$$
\begin{gathered}
\boldsymbol{\beta}^{T}=\left[\begin{array}{llllllllllllllllllll}
1 & 1 & 1 & 0 & 1 & 1 & 1 & 0 & 1 & 1 & 1 & 0 & 1 & 1 & 1 & 0 & 0 & 0 & 0 & 0
\end{array}\right] \\
\boldsymbol{\Sigma}=\left[\begin{array}{llllll}
1.0 & 0.8 & 0.7 & 0.8 \\
0.8 & 1.0 & 0.6 & 0.7 \\
0.7 & 0.6 & 1.0 & 0.8 \\
0.8 & 0.7 & 0.8 & 1.0
\end{array}\right]
\end{gathered}
$$

Scenario $\quad 6: y_{i j k}=\mu+\gamma_{i}+(\gamma \tau)_{i k}+\varepsilon_{i j k} i=1,2,3 ; k=1,2,3,4,5,6 ; j=1, \ldots, n_{i}$ Initial values;

$$
\begin{aligned}
\boldsymbol{\beta}^{T}=\left[\begin{array}{lllllllllllllllllllllllllll}
1 & 1 & 1 & 0 & 1 & 1 & 1 & 1 & 1 & 0 & 1 & 1 & 1 & 1 & 1 & 0 & 1 & 1 & 1 & 1 & 1 & 0 & 0 & 0 & 0 & 0 & 0
\end{array}\right] \\
\boldsymbol{\Sigma}=\left[\begin{array}{llllllll}
1.0 & 0.8 & 0.7 & 0.6 & 0.8 & 0.8 \\
0.8 & 1.0 & 0.6 & 0.8 & 0.7 & 0.8 \\
0.7 & 0.6 & 1.0 & 0.6 & 0.8 & 0.7 \\
0.6 & 0.8 & 0.6 & 1.0 & 0.6 & 0.7 \\
0.8 & 0.7 & 0.8 & 0.6 & 1.0 & 0.8 \\
0.8 & 0.8 & 0.7 & 0.7 & 0.8 & 1.0
\end{array}\right]
\end{aligned}
$$


Table 5. Simulation Results for Scenario 1

\begin{tabular}{|c|c|c|c|c|c|}
\hline$\hat{\boldsymbol{\beta}}$ & $n=20$ & $n=60$ & $n=100$ & $n=140$ & $n=200$ \\
\hline 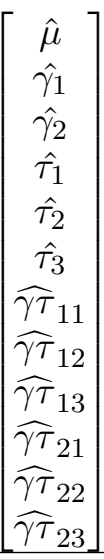 & {$\left[\begin{array}{c}0.9343357 \\
0.4242187 \\
-0.4242187 \\
-0.9377539 \\
-0.9471964 \\
1.8849502 \\
-0.4205610 \\
-0.4289108 \\
0.8549618 \\
0.4260510 \\
0.4289108 \\
-0.8549618\end{array}\right]$} & {$\left[\begin{array}{c}0.9420114 \\
0.4328049 \\
-0.4328049 \\
-0.9412424 \\
-0.9401964 \\
1.8814388 \\
-0.4322294 \\
-0.4317248 \\
0.8639542 \\
0.4322294 \\
0.4317248 \\
-0.8639542\end{array}\right]$} & {$\left[\begin{array}{c}0.9384118 \\
0.4312557 \\
-0.4312557 \\
-0.9420785 \\
-0.9447112 \\
1.8867897 \\
-0.4324817 \\
-0.4332632 \\
0.8657449 \\
0.4324817 \\
0.4332632 \\
-0.8639542\end{array}\right]$} & {$\left[\begin{array}{c}0.9454468 \\
0.4317002 \\
-0.4317002 \\
-0.9299563 \\
-0.9350825 \\
1.8650389 \\
-0.4243207 \\
-0.4290144 \\
0.8563351 \\
0.4273207 \\
0.4290144 \\
-0.8563351\end{array}\right]$} & {$\left[\begin{array}{c}0.9450611 \\
0.4343434 \\
-0.4343434 \\
-0.9522405 \\
-0.9473973 \\
1.8996378 \\
-0.4364057 \\
-0.4350103 \\
0.8717160 \\
0.4367057 \\
0.4350103 \\
-0.8717160\end{array}\right]$} \\
\hline$n$ & \multicolumn{5}{|c|}{$\hat{\Sigma}$} \\
\hline 20 & & {$\left[\begin{array}{l}1.0044318 \\
0.7984512 \\
0.7035253\end{array}\right.$} & $\begin{array}{l}0.7984512 \\
0.9958418 \\
0.5990959\end{array}$ & $\left.\begin{array}{l}0.7035253 \\
0.5990959 \\
1.0645054\end{array}\right]$ & \\
\hline 60 & & {$\left[\begin{array}{l}0.9860640 \\
0.7949169 \\
0.6923261\end{array}\right.$} & $\begin{array}{l}0.7949169 \\
0.9993185 \\
0.5979830\end{array}$ & $\left.\begin{array}{l}0.6923261 \\
0.5979830 \\
1.0579719\end{array}\right]$ & \\
\hline 100 & & {$\left[\begin{array}{l}1.0021265 \\
0.7993710 \\
0.7031587\end{array}\right.$} & $\begin{array}{l}0.7993710 \\
0.9954492 \\
0.6000148\end{array}$ & $\left.\begin{array}{l}0.6923261 \\
0.6000148 \\
1.0670697\end{array}\right]$ & \\
\hline 140 & & {$\left[\begin{array}{l}0.9972212 \\
0.7970500 \\
0.6989386\end{array}\right.$} & $\begin{array}{l}0.7970500 \\
0.9964804 \\
0.6009773\end{array}$ & $\left.\begin{array}{l}0.6989386 \\
0.6009773 \\
1.0646139\end{array}\right]$ & \\
\hline 200 & & {$\left[\begin{array}{l}1.0004959 \\
0.8052401 \\
0.6995259\end{array}\right.$} & $\begin{array}{l}0.8052401 \\
1.0035122 \\
0.5999236\end{array}$ & $\left.\begin{array}{l}0.6995259 \\
0.5999236 \\
1.0566948\end{array}\right]$ & \\
\hline
\end{tabular}

Tables 5-6-7-8-9-10 illustrate the simulation results, which are the mean values of estimates for scenarios, for the sample sizes $n=20,60,100,140$ and 200 . The simulation results suggest that the estimates obtained with ML are close to the initial values of parameter. It can be noted that the total number of parameters to be estimated consists of treatment effect, group effect and treatment x group effect. $\hat{\boldsymbol{\Sigma}}$ is the estimation of the scatter matrix. Diagonal elements in $\hat{\boldsymbol{\Sigma}}$ are expected to be close to 1 . Simulation results show that these values are very close to 1 .

Table 11 displays the mean Euclidian distance values of parameter estimates. It is observed that mean Euclidian distances increase while the sample size increases for some scenarios. For example, in scenario 1 and 2, simulation results are better for small samples $n=20$. For scenario 4 mean Euclidian measures decrease when the sampling sizes increase. It is also probable to suggest the same comment for scenario 5 . The best result for scenario 
Table 6. Simulation Results for Scenario 2

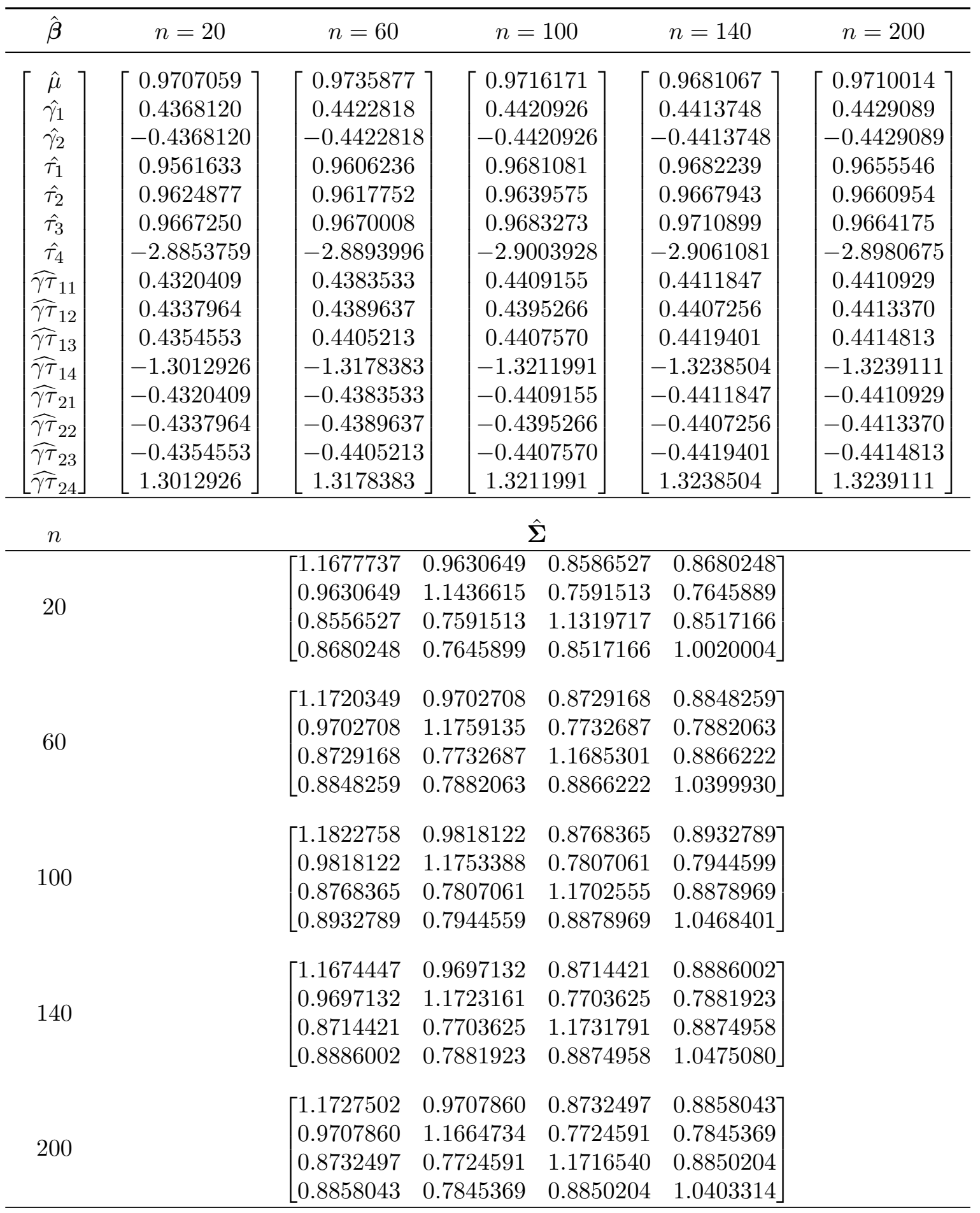

6 occurs with sampling size $n=100$ and 200 for parameter vector $\boldsymbol{\beta}$ estimates. Although parameter estimates generally provide consistent results, in some cases, we have reached the conclusion that the number of samples and dimensions increases as their mean values for mean distance distances increase. We attribute some increases in distances with the number of dimensions in the repeated measures data layout. 
Table 7. Simulation Results for Scenario 3

\begin{tabular}{|c|c|c|c|c|c|c|}
\hline$\hat{\boldsymbol{\beta}}$ & $n=20$ & $n=60$ & \multicolumn{2}{|c|}{$n=100$} & $n=140$ & $n=200$ \\
\hline$\hat{\mu}]$ & {$[0.9836214]$} & {$[0.9824309]$} & \multicolumn{2}{|c|}{$[0.9768839]$} & 0.9739180 & $0.9807626]$ \\
\hline$\hat{\gamma}_{1}$ & 0.4672239 & 0.4707801 & \multirow{2}{*}{\multicolumn{2}{|c|}{$\begin{array}{c}0.4691291 \\
-04691291\end{array}$}} & 0.4684743 & 0.4708221 \\
\hline$\hat{\gamma_{2}}$ & -0.4672239 & -0.4707801 & \multirow{2}{*}{\multicolumn{2}{|c|}{$\begin{array}{c}-0.4691291 \\
0.9785333\end{array}$}} & -0.4684743 & -0.4708221 \\
\hline$\hat{\tau_{1}}$ & 0.9755540 & 0.9784105 & & & 0.9739044 & 0.9783852 \\
\hline$\hat{\tau_{2}}$ & 0.9601416 & 0.9829910 & \multicolumn{2}{|c|}{0.9822740} & 0.9766738 & 0.9746975 \\
\hline$\hat{\tau_{3}}$ & 0.9561368 & 0.9569465 & \multicolumn{2}{|c|}{0.9893693} & 0.9810981 & 0.9803757 \\
\hline$\hat{\tau_{4}}$ & 0.9516076 & 0.9621299 & \multicolumn{2}{|c|}{0.9788247} & 0.9779680 & 0.9788336 \\
\hline$\hat{\tau_{5}}$ & 0.9903658 & 0.9682543 & \multicolumn{2}{|c|}{0.9915044} & 0.9735276 & 0.9867033 \\
\hline$\hat{\tau_{6}}$ & -4.8338057 & -4.8487322 & \multicolumn{2}{|c|}{-4.9205058} & -4.8831719 & -4.8986952 \\
\hline$\widehat{\gamma \tau}_{11}$ & 0.4656464 & 0.4693311 & \multicolumn{2}{|c|}{0.4693910} & 0.4684673 & 0.4698891 \\
\hline$\widehat{\gamma \tau}_{12}$ & 0.4605553 & 0.4709121 & & 0.4693490 & 0.4686269 \\
\hline$\widehat{\gamma \tau}_{13}$ & 0.4560595 & 0.4620782 & \multicolumn{2}{|c|}{0.4730702} & 0.4708738 & 0.4705867 \\
\hline$\widehat{\gamma \tau}_{14}$ & 0.4576254 & 0.4637188 & \multicolumn{2}{|c|}{0.4695142} & 0.4699015 & 0.4699092 \\
\hline$\widehat{\gamma \tau}_{15}$ & 0.4701088 & 0.4659045 & \multicolumn{2}{|c|}{0.4737822} & 0.4683366 & 0.4726697 \\
\hline$\widehat{\gamma \tau}_{16}$ & -2.3129956 & -2.3319447 & \multicolumn{2}{|c|}{-2.3563676} & -2.3469282 & -2.3516816 \\
\hline$\widehat{\gamma \tau}_{21}$ & -0.4656464 & -0.4693311 & \multicolumn{2}{|c|}{-0.4693910} & -0.4684673 & -0.4698891 \\
\hline$\widehat{\gamma \tau}_{22}$ & -0.4605553 & -0.4709121 & \multicolumn{2}{|c|}{-0.4706100} & -0.4693490 & -0.4686269 \\
\hline$\widehat{\gamma \tau}_{23}$ & -0.4590595 & -0.4620782 & \multicolumn{2}{|c|}{-0.4730702} & -0.4708738 & -0.4705867 \\
\hline$\widehat{\gamma \tau}_{24}$ & -0.4576254 & -0.4637188 & \multirow{2}{*}{\multicolumn{2}{|c|}{$\begin{array}{l}-0.4695142 \\
-0.4737822\end{array}$}} & -0.4699015 & -0.4699092 \\
\hline$\widehat{\gamma \tau}_{25}$ & -0.4701088 & -0.4659045 & & & -0.4683366 & -0.4726697 \\
\hline$\left\lfloor\widehat{\gamma \tau}_{26}\right\rfloor$ & 2.3129956 & 2.3319447 & 2.356 & 3676 & 2.3469282 & 2.3516816 \\
\hline$n$ & & & $\hat{\boldsymbol{\Sigma}}$ & is & & \\
\hline & 1.0755235 & 0.8836730 & 0.7874955 & 0.8907883 & 0.7881128 & 0.8416675 \\
\hline & 0.8836730 & 1.0784877 & 0.6997431 & 0.7992564 & 0.6976827 & 0.7473167 \\
\hline 20 & 0.7874955 & 0.6997431 & 1.0757915 & 0.8903538 & 0.7866739 & 0.6571116 \\
\hline 20 & 0.8907883 & 0.7992564 & 0.8903538 & 1.0989230 & 0.6971047 & 0.7560317 \\
\hline & 0.7881128 & 0.6976827 & 0.7866739 & 0.6971047 & 1.0726606 & 0.7505855 \\
\hline & 0.8416675 & 0.7473167 & 0.6571116 & 0.7560317 & 0.7505855 & $1.0199291]$ \\
\hline & Г1.0923928 & 0.8902089 & 0.7901686 & 0.8878455 & 0.7928542 & $0.8393031]$ \\
\hline & 0.8902089 & 1.0845872 & 0.6891677 & 0.7862598 & 0.6874106 & 0.7354355 \\
\hline & 0.7901686 & 0.6891677 & 1.0820087 & 0.8842451 & 0.7882960 & 0.6405009 \\
\hline 60 & 0.8878455 & 0.7862598 & 0.8842451 & 1.0781985 & 0.6878597 & 0.7351337 \\
\hline & 0.7928542 & 0.6874106 & 0.7882960 & 0.6878597 & 1.0933798 & 0.7415198 \\
\hline & 0.8393031 & 0.7354355 & 0.6405009 & 0.7351337 & 0.7415198 & 1.0111390 \\
\hline & {$[1.0981987$} & 0.9010671 & 0.7991361 & 0.9006872 & 0.8018276 & 0.85192627 \\
\hline & 0.9010671 & 1.1050489 & 0.6985855 & 0.8007166 & 0.7048192 & 0.7556434 \\
\hline & 0.7991361 & 0.6985855 & 1.0937092 & 0.8996331 & 0.7989834 & 0.6536867 \\
\hline 100 & 0.9006872 & 0.8007166 & 0.8996331 & 1.0992524 & 0.7048266 & 0.7542599 \\
\hline & 0.8018276 & 0.7048192 & 0.7989834 & 0.7048266 & 1.1003228 & 0.7553096 \\
\hline & 0.8519262 & 0.7556434 & 0.6536867 & 0.7542599 & 0.7553096 & $1.0262999]$ \\
\hline & 1.0929229 & 0.8962434 & 0.7967582 & 0.8988371 & 0.7954586 & 0.84655737 \\
\hline & 0.8962434 & 1.0919103 & 0.6956042 & 0.7984310 & 0.6948726 & 0.7449659 \\
\hline & 0.7967582 & 0.6956042 & 1.0922195 & 0.8958949 & 0.7959612 & 0.6483871 \\
\hline 140 & 0.8988371 & 0.7984310 & 0.8958949 & 1.0962205 & 0.6995046 & 0.7494408 \\
\hline & 0.7954586 & 0.6948726 & 0.7959612 & 0.6995046 & 1.0938862 & 0.7469972 \\
\hline & 0.8465573 & 0.7449659 & 0.6483871 & 0.7494408 & 0.7469972 & $1.0217855]$ \\
\hline & 1.0999886 & 0.9030294 & 0.8001038 & 0.9014575 & 0.8019377 & $0.8522977]$ \\
\hline & 0.9030294 & 1.1028539 & 0.7021088 & 0.8026561 & 0.7030575 & 0.7550096 \\
\hline & 0.8001038 & 0.7021088 & 1.0981524 & 0.9009969 & 0.8004817 & 0.6523121 \\
\hline 200 & 0.9014575 & 0.8026561 & 0.9009969 & 1.1044682 & 0.7009996 & 0.7538706 \\
\hline & 0.8019377 & 0.7030575 & 0.8004817 & 0.7009996 & 1.1004649 & 0.7520755 \\
\hline & 0.8522977 & 0.7550096 & 0.6523121 & 0.7538706 & 0.7520755 & 1.0267292 \\
\hline
\end{tabular}


Table 8. Simulation Results for Scenario 4

\begin{tabular}{|c|c|c|c|c|c|}
\hline$\hat{\boldsymbol{\beta}}$ & $n=20$ & $n=60$ & $n=100$ & $n=140$ & $n=200$ \\
\hline$\hat{\mu}]$ & 0.9366305 & {$[0.9346919]$} & 0.9491782 & 0.9368637 & 0.9327677 \\
\hline$\hat{\gamma_{1}}$ & 0.2766231 & 0.2784529 & 0.2823623 & 0.2797428 & 0.2786060 \\
\hline$\hat{\gamma_{2}}$ & 0.2763619 & 0.2786254 & 0.2823541 & 0.2797428 & 0.2786060 \\
\hline$\hat{\gamma_{3}}$ & -0.5529850 & -0.5570783 & -0.5647163 & -0.5594855 & -0.5572120 \\
\hline$\hat{\tau_{1}}$ & 0.9408612 & 0.9425108 & 0.9302790 & 0.9342348 & 0.9387667 \\
\hline$\hat{\tau_{2}}$ & 0.9404960 & 0.9470248 & 0.9336892 & 0.9283549 & 0.9370950 \\
\hline$\hat{\tau_{3}}$ & -1.8813572 & -1.8895356 & -1.8639682 & -1.8625897 & -1.8758617 \\
\hline$\widehat{\gamma \tau}_{11}$ & 0.2790280 & 0.2801211 & 0.2780030 & 0.2789619 & 0.2801989 \\
\hline$\widehat{\gamma \tau}_{12}$ & 0.2794410 & 0.2809977 & 0.2788274 & 0.2775991 & 0.2798438 \\
\hline$\widehat{\gamma \tau}_{13}$ & -0.5584689 & -0.5611188 & -0.5568305 & -0.5565610 & -0.5600427 \\
\hline$\widehat{\gamma \tau}_{21}$ & 0.2786562 & 0.2802327 & 0.2779960 & 0.2789619 & 0.2801989 \\
\hline$\widehat{\gamma \tau}_{22}$ & 0.2791086 & 0.2811094 & 0.2788190 & 0.2775991 & 0.2798438 \\
\hline$\widehat{\gamma \tau}_{23}$ & -0.5577648 & -0.5613421 & -0.5568151 & -0.5565610 & -0.5600427 \\
\hline$\widehat{\gamma \tau}_{31}^{20}$ & -0.5576841 & 0.5603538 & -0.5559991 & -0.5579239 & -0.5603979 \\
\hline$\widehat{\gamma \tau}_{32}$ & -0.5585496 & -0.5621071 & -0.5576465 & -0.5551981 & -0.5596876 \\
\hline$\left[\widehat{\gamma}_{33}\right]$ & 1.1162337 & 1.1224609 & 1.1136455 & $1.1131220\rfloor$ & $1.1200855]$ \\
\hline$n$ & \multicolumn{5}{|c|}{$\hat{\Sigma}$} \\
\hline \multirow{3}{*}{20} & \multicolumn{5}{|c|}{$\left[\begin{array}{lll}1.502018 & 1.303201 & 0.948636\end{array}\right.$} \\
\hline & & 1.303201 & 1.498953 & 0.841603 & \\
\hline & & 0.948636 & 0.841603 & $1.108061]$ & \\
\hline \multirow{3}{*}{60} & & {$[1.5302728$} & 1.3315924 & $0.9697795]$ & \\
\hline & & 1.3315924 & 1.5326334 & 0.8732823 & \\
\hline & & 0.9697795 & 0.8732823 & $1.1565401]$ & \\
\hline \multirow{3}{*}{100} & & {$[1.5338223$} & 1.3330751 & 0.96296727 & \\
\hline & & 1.330751 & 1.5324607 & 0.8621925 & \\
\hline & & 0.9629672 & 0.8621925 & $1.1324817]$ & \\
\hline \multirow{3}{*}{140} & & {$[1.5513922$} & 1.3483842 & 0.97485997 & \\
\hline & & 1.3483842 & 1.5467587 & 0.8724194 & \\
\hline & & 0.9748599 & 0.8724194 & 1.1356878 & \\
\hline \multirow{3}{*}{200} & & {$[1.5410728$} & 1.3400609 & $0.9716799]$ & \\
\hline & & 1.3400609 & 1.5388626 & 0.8731546 & \\
\hline & & 0.9716799 & 0.8731546 & 1.1337651 & \\
\hline
\end{tabular}

\section{Numerical example}

For illustration purposes, we consider the data set o2cons, which is included in MANOVA.RM. This data set contains measurements of the oxygen consumption of leukocytes in the presence and absence of inactivated staphylococci at three consecutive time points. Due to the study design, both time and staphylococci are sub-plot factors while the treatment (Verum vs. Placebo) is a whole-plot factor [14]. o2cons data do not follow the Multivariate Normal distribution. It will be attempted to estimate the unknown parameters under the assumption MLD. First, an OLS estimator for initial values of the $\boldsymbol{\beta}$ parameter vector is used. The data structure has two groups and three treatment conditions as repeated measures so we used the same initial value for the scatter matrix $\boldsymbol{\Sigma}$ 
Table 9. Simulation Results for Scenario 5

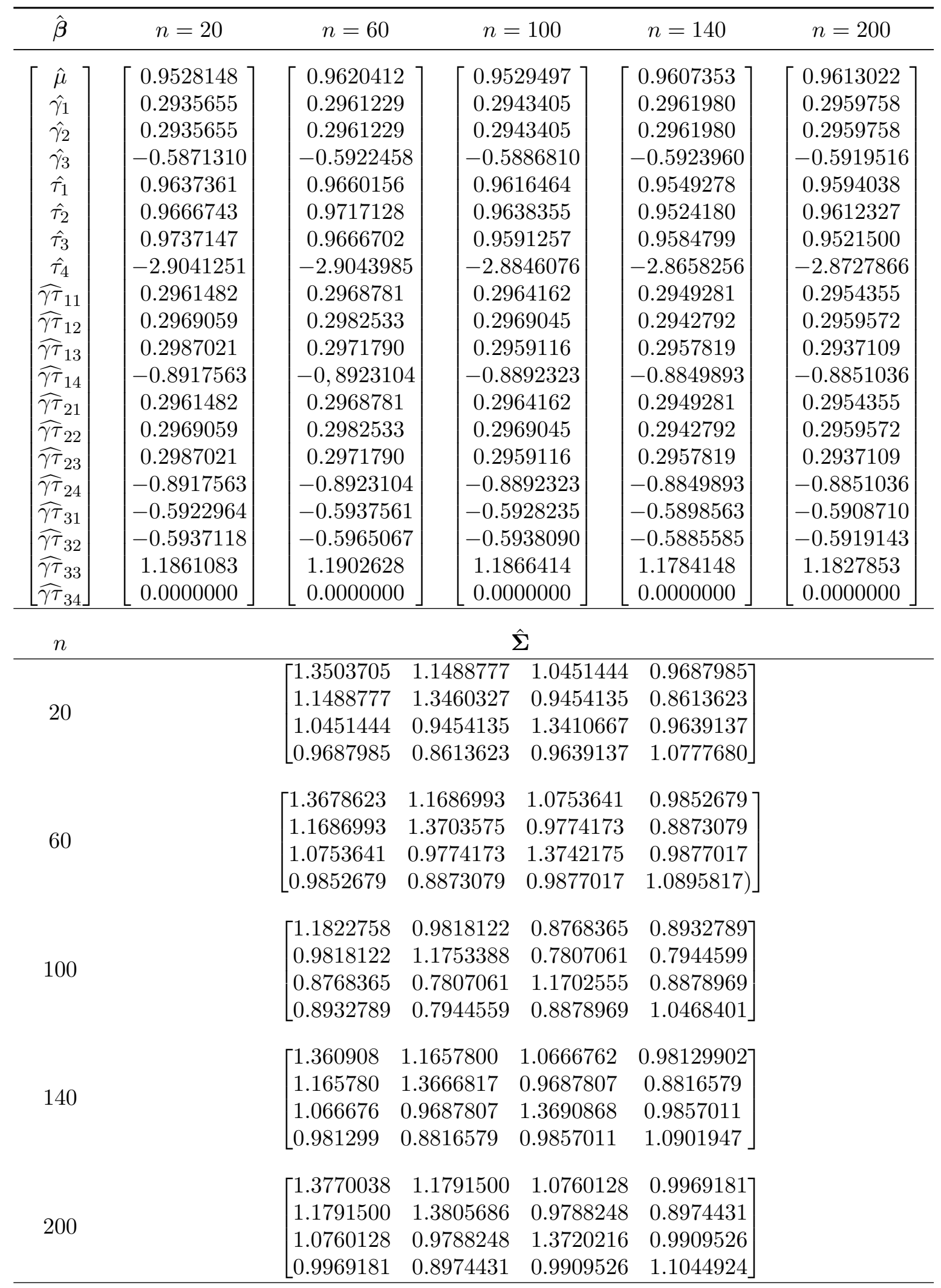


Table 10. Simulation Results for Scenario 6

\begin{tabular}{|c|c|c|c|c|c|c|}
\hline$\hat{\boldsymbol{\beta}}$ & $n=20$ & $n=60$ & \multicolumn{2}{|c|}{$n=100$} & $n=140$ & $n=200$ \\
\hline$\hat{\mu}]$ & {$[1.0028522]$} & 0.9909523 & \multicolumn{2}{|c|}{$[0.9606188]$} & 0.9787644 & $0.9722220^{-}$ \\
\hline$\hat{\gamma_{1}}$ & 0.3198137 & 0.3166943 & \multicolumn{2}{|c|}{0.3087021} & 0.3134651 & 0.3117178 \\
\hline$\hat{\gamma_{2}}$ & 0.3198137 & 0.3166943 & \multicolumn{2}{|c|}{0.3087021} & 0.3134651 & 0.3117178 \\
\hline$\hat{\gamma_{3}}$ & -0.6396273 & -0.6333887 & \multicolumn{2}{|c|}{-0.6174042} & -0.6269302 & -0.6234356 \\
\hline$\hat{\tau_{1}}$ & 0.9674864 & 0.9562702 & \multicolumn{2}{|c|}{0.9752554} & 0.9630041 & 0.9719804 \\
\hline$\hat{\tau_{2}}$ & 0.9801503 & 0.9638313 & \multicolumn{2}{|c|}{0.9751886} & 0.9676519 & 0.9744905 \\
\hline$\hat{\tau_{3}}$ & 0.9582406 & 0.9548903 & \multicolumn{2}{|c|}{0.9690924} & 0.9599203 & 0.9679900 \\
\hline$\hat{\tau_{4}}$ & 0.9731756 & 0.9585004 & \multicolumn{2}{|c|}{0.9742503} & 0.9690610 & 0.9736910 \\
\hline$\hat{\tau_{5}}$ & 0.9625207 & 0.9617886 & \multicolumn{2}{|c|}{0.9732970} & 0.9627918 & 0.9737203 \\
\hline$\hat{\tau}_{6}$ & -4.8415736 & -4.7952808 & \multicolumn{2}{|c|}{-4.8670837} & -4.8224291 & -4.8618723 \\
\hline$\widehat{\gamma \tau}_{11}$ & 0.3108945 & 0.3080250 & \multicolumn{2}{|c|}{0.3125616} & 0.3094894 & 0.3116720 \\
\hline$\widehat{\gamma \tau}_{12}$ & 0.3140003 & 0.3099579 & \multirow{2}{*}{\multicolumn{2}{|c|}{$\begin{array}{l}0.3125231 \\
0.3110277\end{array}$}} & 0.3106156 & 0.3123014 \\
\hline$\widehat{\gamma \tau}_{13}^{12}$ & 0.3089081 & 0.3077432 & & & 0.3086798 & 0.3106648 \\
\hline$\widehat{\gamma \tau}_{14}$ & 0.3123360 & 0.3086346 & \multicolumn{2}{|c|}{0.3123081} & 0.3109668 & 0.3121374 \\
\hline$\widehat{\gamma \tau}_{15}$ & 0.3100063 & 0.3094667 & \multicolumn{2}{|c|}{0.3120308} & 0.3093383 & 0.3121092 \\
\hline$\widehat{\gamma \tau}_{16}$ & -1.5561452 & -1.5438273 & \multicolumn{2}{|c|}{-1.5604514} & -1.5490898 & -1.5588849 \\
\hline$\widehat{\gamma \tau}_{21}^{10}$ & 0.3108945 & 0.3080250 & 0.312 & 25616 & 0.3094894 & 0.3116720 \\
\hline$\widehat{\gamma \tau}_{22}^{21}$ & 0.3140003 & 0.3099579 & 0.312 & 5231 & 0.3106156 & 0.3123014 \\
\hline$\widehat{\gamma \tau}_{23}$ & 0.3089081 & 0.3077432 & 0.311 & 0277 & 0.3086798 & 0.3106648 \\
\hline$\widehat{\gamma \tau}_{24}$ & 0.3123360 & 0.3086346 & 0.312 & 3081 & 0.3109668 & 0.3121374 \\
\hline$\widehat{\gamma \tau}_{25}$ & 0.3100063 & 0.3094667 & 0.312 & 20308 & 0.3093383 & 0.3121092 \\
\hline$\widehat{\gamma \tau}_{26}^{20}$ & -1.5561452 & -1.5438273 & -1.56 & 04514 & -1.5490898 & -1.5588849 \\
\hline$\widehat{\gamma \tau}_{31}$ & -0.6217891 & -0.6160499 & -0.62 & 51233 & -0.6189788 & -0.6233440 \\
\hline$\widehat{\gamma \tau}_{32}$ & -0.6280005 & -0.6199158 & -0.62 & 50462 & -0.6212312 & -0.6246028 \\
\hline$\widehat{\gamma \tau}_{33}$ & -0.6178163 & -0.6154863 & -0.62 & 20553 & -0.6173596 & -0.6213297 \\
\hline$\widehat{\gamma \tau}_{34}$ & -0.6246720 & -0.6172692 & -0.62 & 46162 & -0.6219335 & -0.6242748 \\
\hline$\widehat{\gamma \tau}_{35}$ & -0.6200126 & -0.6189334 & -0.62 & 40617 & -0.6186766 & -0.6242185 \\
\hline$\left[\widehat{\gamma \tau}_{36}\right.$ & 3.1122905 & 3.0876547 & 3.120 & 9027 & 3.0981797 & 3.1177699 \\
\hline$n$ & & & $\hat{\Sigma}$ & $\hat{\hat{~}}$ & & \\
\hline & {$[1.1947156$} & 0.9942241 & 0.9067181 & 0.7968934 & $=1.0002224$ & 0.8866544 \\
\hline & 0.9942241 & 1.1977960 & 0.8009921 & 1.0029247 & 0.9017692 & 0.8872403 \\
\hline & 0.9067181 & 0.8009921 & 1.2199331 & 0.8130626 & 1.0097682 & 0.7947690 \\
\hline 20 & 0.7968934 & 1.0029247 & 0.8130626 & 1.2068645 & 0.8044220 & 0.7927160 \\
\hline & 1.0002224 & 0.9017692 & 1.0097682 & 0.8044220 & 1.2040008 & 0.8910289 \\
\hline & 0.8866544 & 0.8872403 & 0.79447690 & 0.7927160 & 0.8910289 & $1.0299167\rfloor$ \\
\hline & $\lceil 1.2340127$ & 1.0344643 & 0.9324424 & 0.8341462 & 1.0340553 & $0.9171051]$ \\
\hline & 1.0344643 & 1.2335107 & 0.8338232 & 1.0331279 & 0.9352019 & 0.9168444 \\
\hline 60 & 0.9324424 & 0.8338232 & 1.2297561 & 0.8344293 & 1.0312778 & 0.8175206 \\
\hline 60 & 0.8341462 & 1.0331279 & 0.8344293 & 1.2300829 & 0.8363837 & 0.8158956 \\
\hline & 1.0340553 & 0.9352019 & 1.0312778 & 0.8363837 & 1.2345447 & 0.9159340 \\
\hline & 0.9171051 & 0.9168444 & 0.8175206 & 0.8158956 & 0.9159340 & 1.0546494 \\
\hline & {$[1.2266789$} & 1.0245507 & 0.9276357 & 0.8229728 & 1.0237623 & 0.90787027 \\
\hline & 1.0245507 & 1.2200658 & 0.8280308 & 1.0218404 & 0.9266147 & 0.9053670 \\
\hline & 0.9276357 & 0.8280308 & 1.2295837 & 0.8286881 & 1.0297146 & 0.8154763 \\
\hline 100 & 0.8229728 & 1.0218404 & 0.8286881 & 1.2260541 & 0.8268354 & 0.8068379 \\
\hline & 1.0237623 & 0.9266147 & 1.0297146 & 0.8268354 & 1.2260066 & 0.9109103 \\
\hline & 0.9078702 & 0.9053670 & 0.8157563 & 0.8068379 & 0.9109103 & $1.0491001]$ \\
\hline & $\lceil 1.2339762$ & 1.0343395 & 0.9357592 & 0.8339657 & 1.0333046 & $0.9165417]$ \\
\hline & 1.0343395 & 1.2328777 & 0.8326196 & 1.0324785 & 0.9320895 & 0.9156819 \\
\hline 140 & 0.9357592 & 0.8326196 & 1.2328772 & 0.8316858 & 1.0302833 & 0.8172105 \\
\hline 140 & 0.8339657 & 1.0324785 & 0.8316858 & 1.2306429 & 0.8289535 & 0.8134696 \\
\hline & 1.0333046 & 0.9320895 & 1.0302833 & 0.8289535 & 1.2276224 & 0.9140859 \\
\hline & 0.9165417 & 0.9156819 & 0.8172105 & 0.8314696 & 0.9140859 & 1.0557772 \\
\hline & {$[1.2274106$} & 1.0265792 & 0.9297690 & 0.8247520 & 1.0303623 & 0.91469597 \\
\hline & 1.0265792 & 1.2236369 & 0.8305477 & 1.0229057 & 0.9295806 & 0.9121590 \\
\hline 200 & 0.9297690 & 0.8305477 & 1.2256355 & 0.8262686 & 1.0291379 & 0.8156664 \\
\hline 200 & 0.8247520 & 1.0229057 & 0.8262686 & 1.2218103 & 0.8276893 & 0.8095742 \\
\hline & 1.0303623 & 0.9295806 & 1.0291379 & 0.8276893 & 1.2323557 & 0.9174461 \\
\hline & 0.9146959 & 0.9121590 & 0.8156664 & 0.8095742 & 0.9174461 & 1.0587071 \\
\hline
\end{tabular}


Table 11. Mean Euclidian Distance Values of Estimates

\begin{tabular}{|c|c|c|c|c|c|c|}
\hline Scenario & $n$ & $\left\|\hat{\boldsymbol{\beta}}^{k+1}-\hat{\boldsymbol{\beta}}^{k}\right\|$ & $\left\|\hat{\boldsymbol{\Sigma}}^{k+1}-\hat{\boldsymbol{\Sigma}}^{k}\right\|$ & $\left\|\hat{\mathbf{Q}}^{k+1}-\hat{\mathbf{Q}}^{k}\right\|$ & Mean Iteration & Standard Error \\
\hline \multirow{5}{*}{1} & 20 & 0.0035187 & 0.0012888 & 0.0010789 & 21.464 & \pm 0.201577 \\
\hline & 60 & 0.0046643 & 0.0017062 & 0.0000962 & 19.267 & \pm 0.049418 \\
\hline & 100 & 0.0056775 & 0.0019205 & 0.0001710 & 18.996 & \pm 0.044112 \\
\hline & 140 & 0.0051068 & 0.0017600 & 0.0001263 & 18.989 & \pm 0.080891 \\
\hline & 200 & 0.0062360 & 0.0017531 & 0.0001795 & 18.900 & \pm 0.046391 \\
\hline \multirow{5}{*}{2} & 20 & 0.0078829 & 0.0030974 & 0.0004803 & 20.825 & \pm 0.058854 \\
\hline & 60 & 0.0100889 & 0.0024378 & 0.0003827 & 20.274 & \pm 0.035639 \\
\hline & 100 & 0.0105645 & 0.0034435 & 0.0002063 & 20.120 & \pm 0.037215 \\
\hline & 140 & 0.0104771 & 0.0049707 & 0.0002650 & 20.211 & \pm 0.032220 \\
\hline & 200 & 0.0107655 & 0.0031854 & 0.0002447 & 20.248 & \pm 0.031296 \\
\hline \multirow{5}{*}{3} & 20 & 0.0103165 & 0.0037699 & 0.0002837 & 21.299 & \pm 0.030141 \\
\hline & 60 & 0.0171243 & 0.0045007 & 0.0002157 & 20.858 & \pm 0.032877 \\
\hline & 100 & 0.0179484 & 0.0050036 & 0.0001985 & 20.852 & \pm 0.024138 \\
\hline & 140 & 0.0144353 & 0.0054911 & 0.0002194 & 20.875 & \pm 0.021536 \\
\hline & 200 & 0.0184408 & 0.0054787 & 0.0002254 & 20.883 & \pm 0.015670 \\
\hline \multirow{5}{*}{4} & 20 & 0.0108583 & 0.0021096 & 0.0002691 & 21.090 & \pm 0.159654 \\
\hline & 60 & 0.0092014 & 0.0021501 & 0.0002542 & 20.385 & \pm 0.111477 \\
\hline & 100 & 0.0085405 & 0.0037565 & 0.0002331 & 20.441 & \pm 0.122046 \\
\hline & 140 & 0.0084677 & 0.0042293 & 0.0002760 & 20.247 & \pm 0.035117 \\
\hline & 200 & 0.0077615 & 0.0027882 & 0.0002747 & 20.385 & \pm 0.036580 \\
\hline \multirow{5}{*}{5} & 20 & 0.0120570 & 0.0035496 & 0.0004953 & 20.759 & \pm 0.040725 \\
\hline & 60 & 0.0115271 & 0.0035303 & 0.0002265 & 20.447 & \pm 0.038867 \\
\hline & 100 & 0.0114756 & 0.0051898 & 0.0002516 & 20.500 & \pm 0.030967 \\
\hline & 140 & 0.0114183 & 0.0033263 & 0.0002137 & 20.493 & \pm 0.030180 \\
\hline & 200 & 0.0122180 & 0.0036209 & 0.0002228 & 20.509 & \pm 0.025230 \\
\hline \multirow{5}{*}{6} & 20 & 0.0217306 & 0.0066691 & 0.0001968 & 21.385 & \pm 0.034145 \\
\hline & 60 & 0.0245766 & 0.0050620 & 0.0003343 & 21.188 & \pm 0.034026 \\
\hline & 100 & 0.0199006 & 0.0064145 & 0.0002507 & 21.228 & \pm 0.026995 \\
\hline & 140 & 0.0226615 & 0.0052874 & 0.0002118 & 21.235 & \pm 0.024132 \\
\hline & 200 & 0.0187253 & 0.0065136 & 0.0002401 & 21.284 & \pm 0.018321 \\
\hline
\end{tabular}

because the real data structure is the same as in scenario 1 . Initial values is given as follows,

$$
\begin{aligned}
& \hat{\boldsymbol{\beta}}_{\text {OLS }}^{T}=\left[\begin{array}{llllllllllll}
2.57 & 0.12 & -0.12 & -1.42 & -0.70 & 2.10 & -0.80 & -0.40 & 1.06 & 0.71 & 0.351 & -1.07
\end{array}\right] \\
& \boldsymbol{\Sigma}=\left[\begin{array}{lll}
1.0 & 0.8 & 0.7 \\
0.8 & 1.0 & 0.6 \\
0.7 & 0.6 & 1.0
\end{array}\right]
\end{aligned}
$$

In this example, we compute the parameter estimates of Repeated Measures MANOVA model under the assumption MLD. Table 12 illustrates the parameter estimates and Euclidian distances are given in Table 13.

It can be said that the mean differences are quite small as of 22nd iteration.

\section{Conclusions}

In this paper, we have proposed the parameter estimates for Repeated Measures MANOVA under the MLD as an alternative to normal distribution. We used a different Multivariate Repeated Measures ANOVA approach, which was a mixed-method in which Two-way Repeated Measures ANOVA approaches and MANOVA with General Linear Model are considered together. In general, analyzes are performed on Repeated Measures ANOVA or MANOVA problems under the assumption that errors follow the normal 
Table 12. Parameter Estimates for Oxygen consumption of leukocytes data

\begin{tabular}{cc}
\hline$n$ & $\hat{\boldsymbol{\beta}}$ \\
\hline & {$\left[\begin{array}{c}2.3668735 \\
1.2195407 \\
-1.2195407 \\
-1.4167780 \\
-0.6843779 \\
2.1011559 \\
-0.7186707 \\
-0.3502304 \\
1.0689011 \\
0.7186707 \\
0.3502304 \\
-1.0689011\end{array}\right] \quad\left[\begin{array}{llc}0.2558516 & 0.3980161 & 0.5725207 \\
0.3980161 & 0.7801784 & 1.304231 \\
0.5725207 & 1.0304231 & 1.4995878\end{array}\right]$} \\
\hline
\end{tabular}

Table 13. Mean Euclidian Distance Values for Parameter Estimates

\begin{tabular}{ccccc}
\hline$n$ & $\left\|\hat{\boldsymbol{\beta}}^{k+1}-\hat{\boldsymbol{\beta}}^{k}\right\|$ & $\left\|\hat{\boldsymbol{\Sigma}}^{k+1}-\hat{\boldsymbol{\Sigma}}^{k}\right\|$ & $\left\|\hat{\mathbf{Q}}^{k+1}-\hat{\mathbf{Q}}^{k}\right\|$ & Mean Iteration \\
\hline 48 & 0.001134304 & 0.00188000 & 0.00280359 & 22 \\
\hline
\end{tabular}

distribution. In this context, we made inferences based on MLD, which is a member of the Elliptically Contoured distribution family and which has been studied in recent years. Also, parameter estimates of the Repeated Measures MANOVA model were calculated under the proposed MLD assumption. The simulation study was performed to demonstrate the effectiveness of the parameter estimation. The effectiveness of the parameter estimates were indicated by the mean Euclidian distance values and the parameter estimates generally provided consistent results. In the real data example, we have concluded that the mean Euclidian distance values for parameter estimates based on the MLD assumption yield similar results to that of the simulation study. In a conclusion, further studies that aim to improve multivariate test statistics based on parameter estimates that obtain with MLD are recommended for future research.

Acknowledgment. The authors are thankfull to referees for their valuable comments to improve our paper.

\section{References}

[1] T.W. Anderson and K.T. Fang, Theory and Applications of Elliptically Contoured and Related Distributions, Standford,CA, 1990.

[2] T.W. Anderson and K.T. Fang, On The Theory of Multivariate Elliptically Contoured Distributions and Their Applications, Standford,CA, 1992.

[3] O. Arslan, An Alternative Multivariate Skew Laplace distribution: properties and estimation, Statist. Probab. Lett., 51, 865-887, 2010.

[4] O. Arslan, Maximum Likelihood Parameter Estimation for the Multivariate SkewSlash Distribution, Statist. Probab. Lett.,79, 2158-2165, 2009.

[5] O. Arslan and A.I. Genç, A Generalization of the Multivariate Slash Distribution, J. Statist. Plann. Inference, 139, 1164-1170, 2009.

[6] M. Borazan Çelikbıçak, Eliptik Konturlu Dağılımlara Dayalı Çok Değişkenli Tekrarlı Ölçümlü Varyans Analizi, Hacettepe Üniversitesi, Fen Bilimleri Enstitüsü, Basılmamış Doktora Tezi Ankara, 2020. 
[7] Y.M. Bulut, Matris değişkenli Laplace dağılımı: Özellikleri ve parametre tahmini, İstatistikçiler Dergisi: İstatistik ve Aktüerya, 11, 32-41, 2018.

[8] N. Çelik, Anova Modellerinde Carpık Dağılımlar Kullanılarak Dayanıklı Istatistiksel Sonuç Çkarım ve Uygulamaları, Ankara Üniversitesi, Doktora Tezi, 2012.

[9] C.S. Davis, Statistical Methods for the Analysis of Repeated Measurements, 2003.

[10] J.G. Dias and M. Wedel, An empirical comparison of EM, SEM and MCMC performance for problematic Gaussian mixture likelihoods, Stat. Comput., 14, 323-332, 2004.

[11] F.Z. Doğru and O. Arslan, Parameter estimation for mixtures of skew Laplace normal distributions and application in mixture regression modeling, Comm. Statist. Theory Methods, 46, 10879-10896, 2017.

[12] K.T. Fang, S.Kotz, K.W. Ng, Symmetric Multivariate and Related Distributions, 2018.

[13] J. Fox, M. Friendly and S. Weisberg, Hypothesis tests for Multivariate Linear Model Using Car Package, The R Journal, 5, 39-52, 2013.

[14] S. Friedrich, F. Konietschke and M. Pauly, Analysis of Multivariate Data and Repeated Measures Designs with the R Package MANOVA.RM, 2018.

[15] M. Geraci and M.C. Borja, Notebook: The Laplace distribution, Significance, 15, $10-11,2018$.

[16] A.K. Gupta and T. Varga, Elliptically Contoured Models in Statistics, Springer Science and Business Media Dordrecht, 1993.

[17] D.J. Hand and C.C. Taylor, Multivariate Analysis of Variance and Repeated Measures a Practical Approach for Behaviorual Scientists, Chapman and Hall, London, 1987.

[18] S. Kotz, T.J., Kozubowski,and K. Podgórski, The Laplace Distribution and Generalizations, 2001.

[19] T.J. Kozubowski, K., Podgórski and I. Rychlik, , Multivariate Generalized Laplace distribution and Related Random Fields, J. Multivariate Anal., 113, 59-72, 2013.

[20] M. Krzysko,T. Smialowski and W. Wolynski, Analysis of Multivariate Repeated Measures Data using a MANOVA model and Principal Components, Biometrical Letters, 51, 103-114, 2014.

[21] V. Kumar, P. Mehta and G. Shukla, Multivariate Analysis of Repeated Measures Data, 6, 133-148, 2013.

[22] J.K. Lindsey, Multivariate Elliptically Contoured Distributions for Repeated Measurements, Biometrics,55, 1277-1280, 1999.

[23] J.K. Lindsey and P.J. Lindsey, Multivariate Distributions with Correlation Matrices for Nonlinear Repeated Measurements, Comput. Statist. Data Anal., 50, 720-732, 2006.

[24] G. McLachlan and T. Krishnan, The EM Algorithm and Extensions(2nd ed), John Wiley and Sons, Inc., 2008.

[25] D.K. Mcgraw and F. Wagner, Symmetric Distribution, IEEE Trans. Inform. Theory, IT., 14(1), 110-120, 1968.

[26] D.F. Morrison, Multivariate Analysis of Variance, Encyclopedia of Biostatistics, 2005.

[27] S. Nadarajah, The Kotz-Type Distribution with Applications, Statistics (Ber.),37 , 341-358, 2003.

[28] R.G. O'Brien and M.K. Kaiser, MANOVA method for Analyzing Repeated Measures Designs, An Extensive Primer, Psychological bulletin, 97, 316-333, 1985.

[29] K. Plungpongpun, Analysis of Multivariate Data Using Kotz Type Distribution, 2003.

[30] H. Visk, On the Parameter Estimation of the Asymmetric Multivariate Laplace Distribution, Comm. Statist. Theory Methods, 38 ,461-470, 2009.

[31] F.G. Yavuz and O. Arslan, Linear mixed model with Laplace distribution (LLMM), Statist. Papers, 59, 271-289, 2018. 